\title{
Methylene blue injection into the rectal artery as a simple method to improve lymph node harvest in rectal cancer
}

\author{
Bruno Märkl ${ }^{1}$, Therese G Kerwel², Theodor Wagner ${ }^{1}$, Matthias Anthuber ${ }^{2}$ and \\ Hans M Arnholdt ${ }^{1}$ \\ ${ }^{1}$ Institute of Pathology, Klinikum Augsburg, Augsburg, Germany and ${ }^{2}$ Department of Visceral Surgery, \\ Klinikum Augsburg, Augsburg, Germany
}

\begin{abstract}
Adequate lymph node assessment in colorectal cancer is crucial for prognosis estimation and further therapy stratification. However, there is still an ongoing debate on required minimum lymph node numbers and the necessity of advanced techniques such as immunohistochemistry or PCR. It has been proven in several studies that lymph node harvest is often inadequate under routine analysis. Lymph nodes smaller than $5 \mathrm{~mm}$ are especially concerning as they can carry the majority of metastases. These small, but affected lymph nodes may escape detection in routine analysis. Therefore, fat-clearing protocols and sentinel techniques have been developed to improve accuracy of lymph node staging. We describe a novel and simple method of ex vivo methylene blue injection into the superior rectal artery of rectal cancer specimens, which highlights lymph nodes and makes them easy to detect during manual dissection. Initially, this method was developed for proving accuracy of total mesorectal excision. We performed a retrospective study comparing lymph node recovery of 12 methylene blue stained and an equal number of unstained cases. Lymph node recovery differed significantly with average lymph node numbers of $27 \pm 7$ and $14 \pm 4(P<0.001)$ for the methylene blue and the unstained group, respectively. The largest difference was found in size groups between 1 and $4 \mathrm{~mm}$ causing a shift in size distribution toward smaller nodes. Metastases were confirmed in 21 and 19 lymph nodes occurring in five and four cases, respectively. Hence, we conclude that methylene blue injection technique improves accuracy of lymph node staging by heightening the lymph node harvest in rectal resections. In our experience, it is a very simple time and cost effective method that can be easily established under routine circumstances. Modern Pathology (2007) 20, 797-801; doi:10.1038/modpathol.3800824; published online 4 May 2007
\end{abstract}

Keywords: rectal cancer; lymph node; methylene blue; mesorectal excision; staging

Lymph node assessment is an essential part of staging in colorectal cancer. As TNM stage correlates with survival rates in colorectal cancer, it is crucial for prognosis estimation. ${ }^{1}$ Moreover, in many cases which are not treated with preoperative radiation or chemoradiotherapy the decision for or against an adjuvant chemotherapy is made by the lymph node status. $^{2-4}$ The number of 12 identified lymph nodes is defined as a minimum for an adequate assessment by the UICC, ${ }^{5}$ although recommendations published in the past differ considerably in a range from 9 to 18 lymph nodes. ${ }^{6-8}$ It is well known that even the smallest nodes with diameters less than $1 \mathrm{~mm}$ may bear metastases. Therefore, a broad spectrum of

Correspondence: Dr B Märkl, MD, Institute of Pathology, Klinikum Augsburg, Stenglinstr. 2, 86156 Augsburg, Germany. E-mail: Bruno.Maerkl@klinikum-augsburg.de

Received 27 February 2007; revised and accepted 10 April 2007; published online 4 May 2007 techniques beginning with fat clearance methods to sentinel techniques has been introduced in order to increase lymph node counts and improve accuracy. ${ }^{9-11}$

Nevertheless, dissection of lymph nodes in colorectal specimens is often time consuming and especially in cases of neoadjuvant radiotherapy frustrating. Increasing age, American Society of Anesthesiology grade, and preoperative radiotherapy are found to be factors for reduced lymph node harvest. Transverse colectomy and abdominoperineal resected rectal specimens are the resections that show the lowest numbers of detected lymph nodes. ${ }^{12}$

Recently, during routine dissection of rectal resections we found that methylene blue injection into the superior rectal artery might improve the lymph node yield. The methylene blue injection was initially performed in order to assess the accuracy of the total mesorectal excision specimens, 
as recommended by Hermanek et al. ${ }^{13,14}$ Immediately after the injection, it can be judged whether the mesorectal fascia is intact or not. Even small defects are revealed by leakage of the blue dye on the surface of specimen. In the opposite, no outflow indicates a perfect dissection of the specimen.

Surprisingly, this technique does not only highlight possible defects of the mesorectal fascia but also virtually all lymph nodes of the specimens, which are stained blue after formalin fixing and therefore easy to detect.

In this retrospective study, we compared the lymph node harvest of 12 cases after methylene staining with an equal number of cases without methylene injection. The cases of the control group were chosen randomly out of our records during the same time period and the pathological assessment was performed according to our routine protocol by manual technique.

\section{Materials and methods}

Postoperatively, the superior rectal arteries of 12 unopened resections were cannulated with a standard 17-20-G iv-catheter by the surgeon who is using this technique in order to check the integrity of the total mesorectal dissection. After that, the arteries were filled with $15-20 \mathrm{ml}$ of methylene blue solution (50 mg diluted with $0.9 \%$ saline; ratio $1: 3$ ). Subsequently, after partial opening of the specimens they are fixed in $10 \%$ buffered formalin for at least $24 \mathrm{~h}$. Then, beginning from the aboral end of the resections transverse $5-7 \mathrm{~mm}$ thick slices were cut till the end of the tumor regions were reached. Representative areas were embedded using the whole mount technique. The fat of the remaining parts was then dissected and examined by palpation. The fatty tissue was then sliced and stretched in order to reach thin layers and transparency. The cut surfaces were screened for lymph nodes visible as clearly definable light blue round to oval nodes (Figure 1). They can be detected even when they are covered by a thin layer of fat. After paraffin embedding $3 \mu \mathrm{m}$ sections were cut and stained with hematoxylin and eosin. Slides were then examined carefully for the occurrence of metastases. Lymph node diameters were measured using a digital camera with a calibrated software system (Progress C10, Jenoptik, Jena, Germany).

For statistical analysis, the diameters of the lymph nodes were grouped into four categories $(\leq 1,1-2$, $2-4$ and $>4 \mathrm{~mm}$ ) and percentage values based on the total number of lymph nodes were calculated.

A collection of 12 rectal cancer cases where methylene blue was not used were randomly chosen out of our records during the same time period to serve as a control group. Lymph node numbers were compared using the unpaired $t$-test. Percentage values were compared after arcus sinus transformation employing unpaired $t$-test or Mann-Whitney rank sum test depending on the results of the normality test. All data were processed using SigmaStat-Software (Systat Software Inc., Richmond, CA, USA). A probability value of $<0.05$ was considered significant.

\section{Results}

The two groups were well balanced (Table 1). There were no significant differences regarding age, female:male ratio and mean length of the specimens. The methylene group had one less case of preoperative radiation therapy. Concerning pT-classification, it included one case more with a complete remission (ypT 0) and one case less in the pT 3 category.
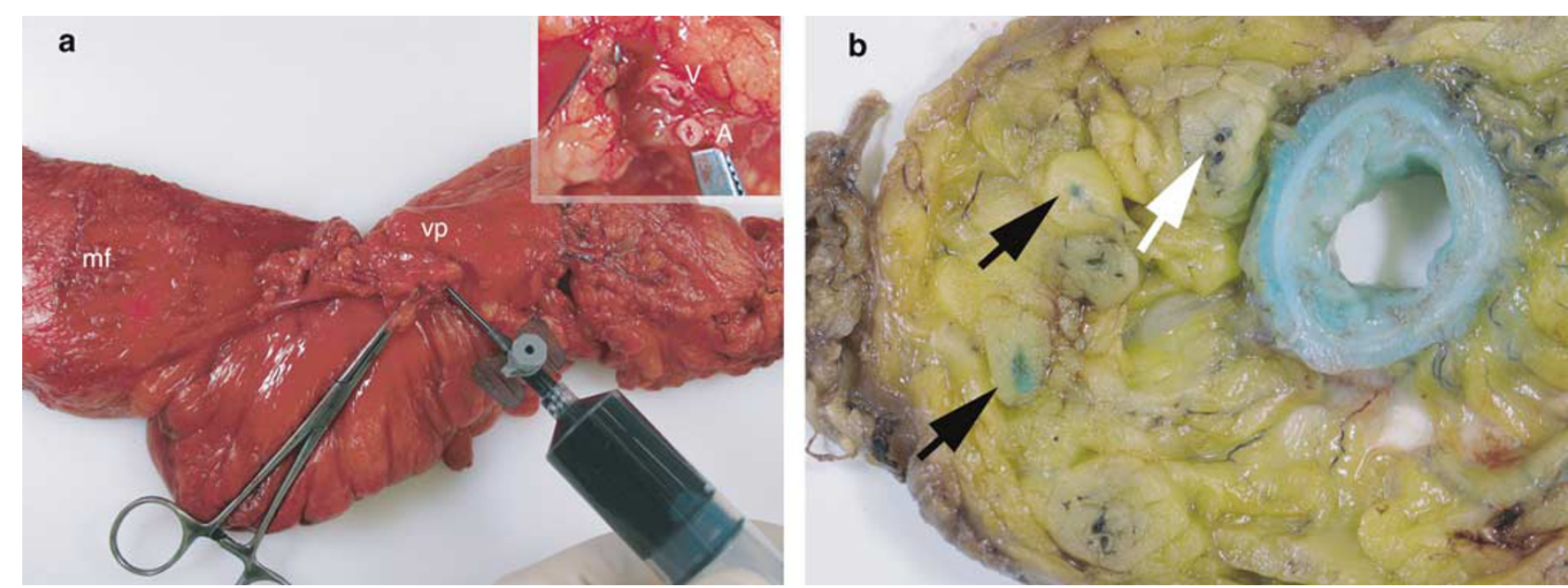

Figure 1 Ex vivo methylene blue injection into superior rectal artery of a rectal resection. $\mathrm{mf}=$ mesorectal fascia; vp=vascular pedicle. Insert: superior rectal artery (A) and its corresponding vene (V). (a) Cross-section and blue staining of two lymph nodes measuring 1 and $3 \mathrm{~mm}$ (black arrow). In contrast, small blood vessels (white arrow) are of dark red-brown color and easy to distinguish from lymph nodes (b). 
To reach an acceptable minimum number of 10 nodes, an additional embedding of vessel-rich pararectal fat was necessary in seven cases of the unstained group. In contrast to that in the methylene group, additional fat embedding was not required (Table 2). Comparison of the mean lymph node harvest showed a highly significant difference between methylene blue-stained and unstained resections with average lymph node numbers of $27 \pm 7$ and $14 \pm 4$, respectively $(P<0.001)$ (Figure 2$)$. The total number of detected lymph nodes was 319 and 173, respectively. Most examined lymph nodes measured between 2 and $4 \mathrm{~mm}$ with proportions of 43,45 and $46 \%$ in the methylene group and the unstained group after and before additional fat embedding (Figure 3). The largest difference between the stained and unstained cases occurred in the size groups of 1-2 and 2-4 mm, causing a shift in size distribution toward smaller nodes (Figure 4). Compared to the unstained group after additional fat embedding, the methylene group was associated with a factor 2.4 and 1.8 higher lymph node harvest in the size groups of 1-2 and 2-4 mm, respectively. Metastases were found in 21 and 19 lymph nodes

Table 1 Group characteristics

\begin{tabular}{lcc}
\hline & $\begin{array}{c}\text { Unstained } \\
\text { group }\end{array}$ & $\begin{array}{c}\text { Methylene } \\
\text { group }\end{array}$ \\
\hline Mean age \pm s.d. & $64 \pm 16$ & $57 \pm 16$ \\
f:m ratio & $1: 1$ & $1: 0.85$ \\
Mean resection length \pm s.d. & $22 \pm 5$ & $20 \pm 4$ \\
Preoperative radiation & 6 & 5 \\
pT0 (complete remission) & 1 & 2 \\
pT2 & 4 & 4 \\
pT3 & 7 & 6 \\
\hline
\end{tabular}

s.d. = standard deviation; $\mathrm{f}=$ female; $\mathrm{m}=$ male. occurring in five and four cases, respectively. Isolated tumor cells were not detected.

\section{Discussion}

Although many published studies have tried to establish a minimal number of examined lymph nodes, it is still not clear if there exists an absolute number that provides a gold standard under a routine analysis. ${ }^{6-8}$ Nevertheless, it seems obvious that accuracy of lymph node status increases with the number of examined lymph nodes. Additionally, Swanson et $a l^{15}$ showed a linear correlation between the number of lymph nodes examined and 5-yearsurvival rates in T3N0 colon cancers. The survival rates ranged from 66.3 to $88.1 \%$. Similar associations were found by Cianchi et $a 7^{7}$ and Goldstein. ${ }^{8}$ In addition, Goldstein calculated a minimum of 30 lymph nodes to reach a probability of $80 \%$ to

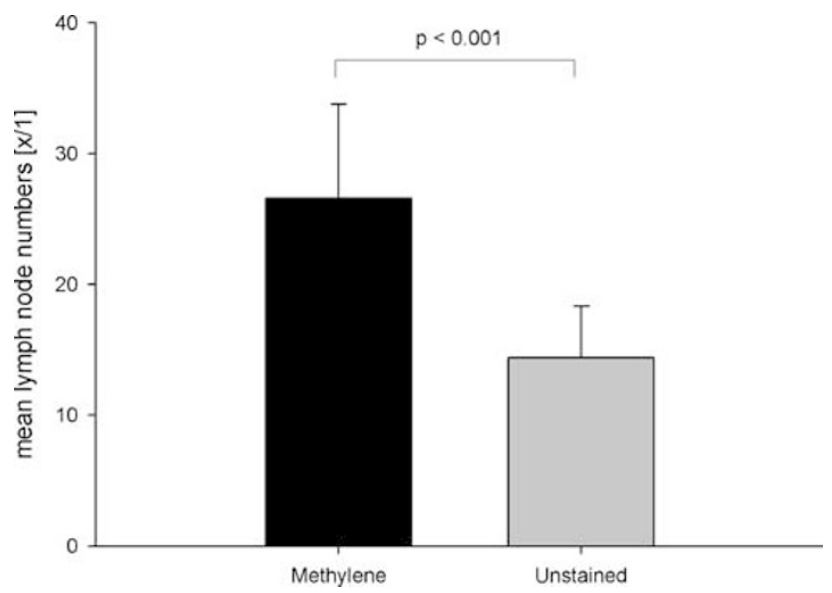

Figure 2 Mean lymph node numbers of the methylene and the unstained group.

Table $2 \mathrm{pN}$ stage and number of collected and positive lymph nodes

\begin{tabular}{|c|c|c|c|c|}
\hline Case & $\begin{array}{c}\text { Methylene group } \\
p N\end{array}$ & $\begin{array}{c}\text { Methylene group Number of } \\
\text { nodes (total positive nodes/ } \\
\text { micrometases) }\end{array}$ & $\begin{array}{l}\text { Unstained group } \\
\qquad N\end{array}$ & $\begin{array}{l}\text { Unstained group Number of } \\
\text { nodes (total positive nodes/ } \\
\text { micrometases) }\end{array}$ \\
\hline 1 & 0 & 18 & 2 & $24(8 / 2)$ \\
\hline 2 & (y) 0 & 18 & (y) 1 & $15(1 / 1)^{\mathrm{a}}$ \\
\hline 3 & (y) 2 & $18(5 / 0)$ & 0 & $11^{\mathrm{a}}$ \\
\hline 4 & (y) 0 & 19 & (у) 0 & 17 \\
\hline 5 & 1 & $32(3 / 1)$ & (y) 0 & 18 \\
\hline 6 & 0 & 39 & 2 & $12(9 / 3)$ \\
\hline 7 & (y) 0 & 28 & (y) 0 & 15 \\
\hline 8 & 0 & 21 & 0 & $14^{\mathrm{b}}$ \\
\hline 9 & 2 & $31(5 / 0)$ & (y) 0 & $11^{\mathrm{b}}$ \\
\hline 10 & 2 & $31(7 / 0)$ & 1 & $11(1 / 1)$ \\
\hline 11 & 1 & $32(1 / 0)$ & (y) 0 & $13^{\mathrm{a}}$ \\
\hline 12 & (y) 0 & 31 & 0 & $11^{\mathrm{a}}$ \\
\hline Total & & 319 (21/1) & & $173(19 / 7)$ \\
\hline
\end{tabular}

$(y)=$ neoadjuvant radiation.

${ }^{a}$ Additional embedding of fat.

${ }^{\mathrm{b}}$ Secondary embedding of additional fat. 
800

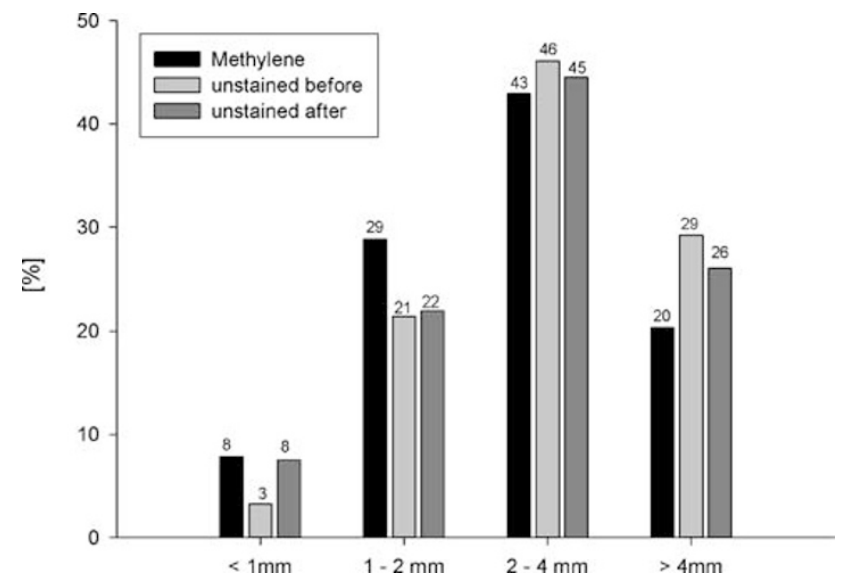

Figure 3 Lymph node size distribution in \% of complete lymph node harvest of 12 methylene blue stained rectal resections and 12 unstained specimens before and after additional fat embedding.

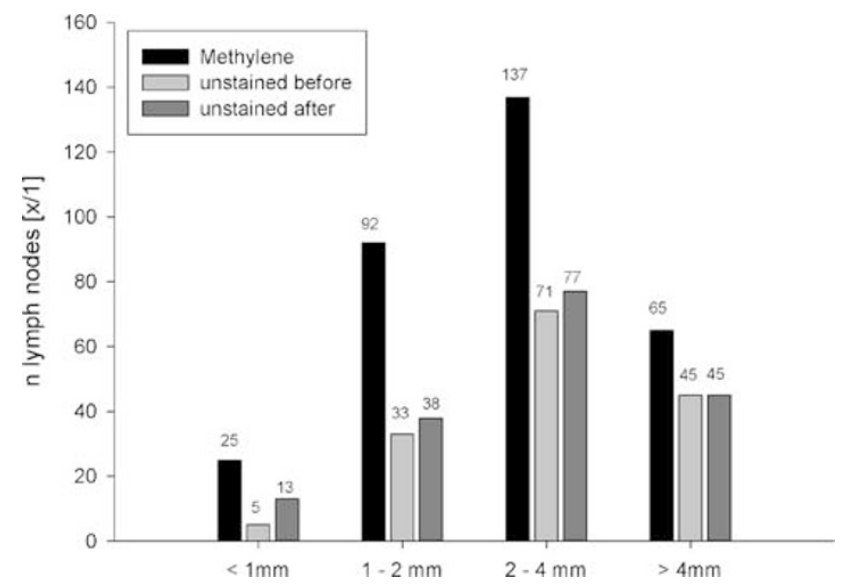

Figure 4 Lymph node size distributions in total numbers of complete lymph node harvest of 12 methylene blue stained rectal resections and 12 unstained specimens before and after additional fat embedding.

identify a single metastasis in a hypothetical specimen. In a study by Scott and Grace, ${ }^{16} 5(8 \%)$ out of 58 cases were upstaged from Dukes B to C after advanced preparation using fat clearance. Haboubi et $a l^{17}$ performed a study in which initially 3 out of 22 cases were upstaged after fat clearance and finally another nine cases turned from Dukes B to $\mathrm{C}$ after employing immunohistochemical staining for cytokeratins. Both studies demonstrated an impressive shift in tumor stage, although it has to be mentioned that initial lymph node harvest was low. Fat clearance methods are an effective way to improve lymph node recovery. Despite that, many of these protocols are difficult and time consuming. Even more problematic is the additional necessity of poisonous chemicals that need to be handled under a hood. Both are circumstances that limit their widespread use. Another advanced technique, the application of immunohistochemical staining methods for the detection of micrometastes is expensive and failed to prove a significant difference concerning 3-year disease-free survival in a meta-analysis performed by Iddings et al. ${ }^{18}$ In contrast confirmation of micrometastes using RT-PCR showed a significant smaller 3-year disease-free survival and reduced overall survival in positive cases.

A different approach to increase accuracy of lymph node staging is the sentinel technique. In vivo and ex vivo methods have been developed, but it seems necessary to combine this technique with advanced methods like immunohistochemistry or PCR to demonstrate an advantage in comparison to conventional dissection. ${ }^{19-21}$ Therefore, it is questionable if these interesting techniques will find usage beyond specialized centers.

In this study, we describe a new and very simple technique to stain lymph nodes of rectal specimens' by ex vivo methylene blue injection into the superior rectal artery making them very easy to detect by conventional dissection. We enrolled 12 cases where this technique was applied (methylene group) and compared them with 12 conventional processed cases (unstained group). Limitations of this study are relatively small case numbers and a retrospective design. Nevertheless, we have demonstrated a highly significant improvement in lymph node harvest (Figure 2) in the methylene group. The main lymph node harvest in this group was $27 \pm 7$ and $86 \%$ higher in comparison to the unstained group. This seems to be even more remarkable when taking into account that the average number of collected nodes in the unstained group is $14 \pm 4$, which is clearly above mean values reported in the literature for conventional techniques., ${ }^{6,12,22}$ Fat clearance methods, or complete fat embedding, produced lymph node numbers between 55 and $87^{10,17,23}$ and showed increased identification of positive nodes. However, these techniques are time consuming and laboring. Almost $50 \%$ of the additional lymph nodes found by Brown et al measured less than $1 \mathrm{~mm}$. But only two out of these 496 very small lymph nodes were metastasized. Most positive lymph nodes showed a size between 1 and $4 \mathrm{~mm}$. This is consistent with findings of other studies. ${ }^{24}$ The largest increase in absolute lymph node numbers in our study was found exactly in these interesting size groups of 1-2 and 2-4 mm (Figure 4). It is worth to emphasizing that this gain is the effect of a very small effort for methylene blue injection into the superior rectal artery, which has furthermore the advantage of proving the accuracy of the mesorectal excision. ${ }^{13,14}$ Whereas additional embedding of mesenteric tissue was necessary in seven cases of the unstained group, no fat embedding was required in the methylene group, which even means an effort reduction. Instead of performing the injection in the operating room, it is also possible to send the resection unfixed to the pathology laboratory, where the injection could be combined with frozen sections of the specimen's distal resection margins. 
The sample size of this study is too small to expect significant differences concerning occurrence of lymph node metastases. A larger prospect study combined with fat clearance or complete submission of mesentery would be necessary. Nevertheless, we have demonstrated that methylene injection technique is a very simple method to improve lymph node harvest in rectal specimens guaranteeing an adequate lymph node assessment. Hence, we can recommend it for routine usage in rectal specimens. Further investigations are necessary to assess if this method can be adapted to other parts of the colon.

\section{Disclosure/conflict of interest}

The authors declare that no conflict of interest exists.

\section{References}

1 Compton CC. Colorectal carcinoma: diagnostic, prognostic, and molecular features. Mod Pathol 2003; 16:376-388.

2 Investigators IB. Efficacy of adjuvant fluorouracil and folinic acid in B2 colon cancer. International Multicentre Pooled Analysis of B2 Colon Cancer Trials (IMPACT B2) Investigators. J Clin Oncol 1999;17:1356-1363.

3 Colorectal Cancer Collaborative Group. Adjuvant radiotherapy for rectal cancer: a systematic overview of 8507 patients from 22 randomised trials. Lancet 2001;358:1291-1304.

4 Sauer R, Becker H, Hohenberger W, et al. Preoperative versus postoperative chemoradiotherapy for rectal cancer. N Engl J Med 2004;351:1731-1740.

5 Sobin LH, Wittekind C. TNM Classification of Malignant Tumors, 6th edn. John Wiley \& Sons: Hoboken, 2002.

6 Maurel J, Launoy G, Grosclaude P, et al. Lymph node harvest reporting in patients with carcinoma of the large bowel: a French population-based study. Cancer 1998;82:1482-1486.

7 Cianchi F, Palomba A, Boddi V, et al. Lymph node recovery from colorectal tumor specimens: recommendation for a minimum number of lymph nodes to be examined. World J Surg 2002;26:384-389.

8 Goldstein NS. Lymph node recoveries from 2427 pT3 colorectal resection specimens spanning 45 years: recommendations for a minimum number of recovered lymph nodes based on predictive probabilities. Am J Surg Pathol 2002;26:179-189.

9 Koren R, Kyzer S, Paz A, et al. Lymph node revealing solution: a new method for detection of minute axillary lymph nodes in breast cancer specimens. Am J Surg Pathol 1997;21:1387-1390.
10 Brown HG, Luckasevic TM, Medich DS, et al. Efficacy of manual dissection of lymph nodes in colon cancer resections. Mod Pathol 2004;17:402-406.

11 Smith J, Hwang H, Wiseman KW, et al. Ex vivo sentinel lymph node mapping in colon cancer: improving the accuracy of pathologic staging? Am J Surg 2006; 191:665-668.

12 Tekkis PP, Smith JJ, Heriot AG, et al. A national study on lymph node retrieval in resectional surgery for colorectal cancer. Dis Colon Rectum 2006;49: 1673-1683.

13 Hermanek $\mathrm{P}$, Hermanek $\mathrm{P}$, Hohenberger $\mathrm{W}$, et al. The pathological assessment of mesorectal excision: implications for further treatment and quality management. Int J Colorectal Dis 2003;18:335-341.

14 Sterk P, Nagel T, Gunter S, et al. Methods for postoperative evaluation of complete excision of the mesorectum. Zentralbl Chir 2000;125:370-374.

15 Swanson RS, Compton CC, Stewart AK, et al. The prognosis of T3No colon cancer is dependent on the number of lymph nodes examined. Ann Surg Oncol 2003;10:65-71.

16 Scott KW, Grace RH. Detection of lymph node metastases in colorectal carcinoma before and after fat clearance. Br J Surg 1989;76:1165-1167.

17 Haboubi NY, Clark P, Kaftan SM, et al. The importance of combining xylene clearance and immunohistochemistry in the accurate staging of colorectal carcinoma. J R Soc Med 1992;85:386-388.

18 Iddings D, Ahmad A, Elashoff D, et al. The prognostic effect of micrometastases in previously staged lymph node negative (No) colorectal carcinoma: A metaanalysis. Ann Surg Oncol 2006;13:1386-1392.

19 Wong JH, Steineman S, Calderia C, et al. Ex vivo sentinel node mapping in carcinoma of the colon and rectum. Ann Surg 2001;233:515-521.

20 Wong JH, Johnson DS, Namiki T, et al. Validation of ex vivo lymphatic mapping in hematoxylin-eosin nodenegative carcinoma of the colon and rectum. Ann Surg Oncol 2004;11:772-777.

21 Braat AE, Oosterhuis JW, de Vries JE, et al. Lymphatic staging in colorectal cancer: pathologic, molecular, and sentinel node techniques. Dis Colon Rectum 2005; 48:371-383.

22 Johnson PM, Malatjalian D, Porter GA. Adequacy of nodal harvest in colorectal cancer: a consecutive cohort study. J Gastrointest Surg 2002;6:883-888; discussion 9-90.

23 Haboubi NY, Abdalla SA, Amini S, et al. The novel combination of fat clearance and immunohistochemistry improves prediction of the outcome of patients with colorectal carcinomas: a preliminary study. Int J Colorectal Dis 1998;13:99-102.

24 Herrera L, Villarreal JR. Incidence of metastases from rectal adenocarcinoma in small lymph nodes detected by a clearing technique. Dis Colon Rectum 1992;35: 783-788. 